The University of San Francisco

USF Scholarship: a digital repository @ Gleeson Library |

Geschke Center

2012

\title{
Rare-Earth-Doped Materials with Application to Optical Signal Processing, Quantum Information Science, and Medical Imaging Technology
}

\author{
R L. Cone
}

CW. Thiel

Y Sun

Thomas Böttger

University of San Francisco, tbottger@usfca.edu

R M. MacFarlane

Follow this and additional works at: http://repository.usfca.edu/phys

Part of the Physics Commons

\section{Recommended Citation}

R. L. Cone ; C. W. Thiel ; Y. Sun ; Thomas Böttger and R. M. Macfarlane "Rare-earth-doped materials with application to optical signal processing, quantum information science, and medical imaging technology", Proc. SPIE 8272, Advances in Photonics of Quantum Computing, Memory, and Communication V, 82720E (February 9, 2012); doi:10.1117/12.909154 


\title{
Rare-earth-doped materials with application to optical signal processing, quantum information science, and medical imaging technology
}

\author{
R. L. Cone, ${ }^{* a}$ C. W. Thiel,,${ }^{a}$ Y. Sun, ${ }^{c, a}$ Thomas Böttger, ${ }^{\text {, a a and R. M. Macfarlane }}{ }^{a, e}$ \\ ${ }^{a}$ Department of Physics, Montana State University, Bozeman, MT 59717; \\ ${ }^{\mathrm{b}}$ Spectrum Lab, Montana State University, Bozeman, MT 59717; \\ ${ }^{c}$ Department of Physics, The University of South Dakota, Vermillion, SD 57069; \\ ${ }^{\mathrm{d}}$ Department of Physics, University of San Francisco, 2130 Fulton Street, San Francisco, CA 94117; \\ ${ }^{\mathrm{e}}$ IBM Almaden Research Center, 650 Harry Road, San Jose, CA 95120
}

\begin{abstract}
Unique spectroscopic properties of isolated rare earth ions in solids offer optical linewidths rivaling those of trapped single atoms and enable a variety of recent applications. We design rare-earth-doped crystals, ceramics, and fibers with persistent or transient "spectral hole" recording properties for applications including high-bandwidth optical signal processing where light and our solids replace the high-bandwidth portion of the electronics; quantum cryptography and information science including the goal of storage and recall of single photons; and medical imaging technology for the 700-900 nm therapeutic window. Ease of optically manipulating rare-earth ions in solids enables capturing complex spectral information in $10^{5}$ to $10^{8}$ frequency bins. Combining spatial holography and spectral hole burning provides a capability for processing high-bandwidth RF and optical signals with sub-MHz spectral resolution and bandwidths of tens to hundreds of $\mathrm{GHz}$ for applications including range-Doppler radar and high bandwidth $\mathrm{RF}$ spectral analysis. Simply stated, one can think of these crystals as holographic recording media capable of distinguishing up to $10^{8}$ different colors. Ultra-narrow spectral holes also serve as a vibration-insensitive sub-kHz frequency reference for laser frequency stabilization to a part in $10^{13}$ over tens of milliseconds. The unusual properties and applications of spectral hole burning of rare earth ions in optical materials are reviewed. Experimental results on the promising $\mathrm{Tm}^{3+}: \mathrm{LiNbO}_{3}$ material system are presented and discussed for medical imaging applications. Finally, a new application of these materials as dynamic optical filters for laser noise suppression is discussed along with experimental demonstrations and theoretical modeling of the process.
\end{abstract}

Keywords: UOT, hole burning, quantum memory, rare earth, medical imaging, laser frequency stabilization, laser phase noise, signal processing

\section{INTRODUCTION}

Rare earth materials have special properties making them ideal for resonant laser-excited optical processing devices. This paper focuses on new applications made practical by exploiting the detailed nature of the optical transition lineshapes at low temperature. These optical transitions provide the basis for optical technology in which light dynamically interacts with a resonant optical material. Applications span from classical signal processing to laser frequency stabilization to quantum memories to filters for ultrasound optical tomography (UOT). We briefly review a number of these technologies and present experimental measurements on $0.1 \% \mathrm{Tm}^{3+}$ : $\mathrm{LiNbO}_{3}$, demonstrating material properties well-suited for high-performance optical filtering in new medical imaging technologies. We also review a new application of these materials as dynamic optical filters for laser phase noise suppression that we have recently reported. ${ }^{1}$ Experimental measurements of this laser spectrum narrowing effect are presented and discussed along with numerical modeling of the results. These models are also used to demonstrate how this novel effect may be simply implemented to produce sub-megahertz high contrast optical filters with very sharp spectral edges.

*cone@montana.edu; phone 1406994 6175; fax 1406994 4452; http://www.physics.montana.edu/faculty/cone/

Advances in Photonics of Quantum Computing, Memory, and Communication V, edited by Zameer UI Hasan, Philip R. Hemmer, Hwang Lee, Charles M. Santori, Proc. of SPIE Vol. 8272, 82720E · @ 2012 SPIE · CCC code: 0277-786X/12/\$18 - doi: 10.1117/12.909154 


\section{BASIC ASPECTS OF RARE EARTH IONS IN SOLIDS}

Only transition elements form stable compounds with partially filled electron shells that are required for localized electronic transitions and sharp lines. These include transition metals $\left(3 \mathrm{~d}^{\mathrm{N}}, 4 \mathrm{~d}^{\mathrm{N}}\right.$, or $\left.5 \mathrm{~d}^{\mathrm{N}}\right)$, rare earths (also known as lanthanides) $\left(4 \mathrm{f}^{\mathrm{N}}\right)$, and actinides $\left(5 \mathrm{f}^{\mathrm{N}}\right)$. The partially filled $4 \mathrm{f}^{\mathrm{N}}$ shells of rare earth ions give useful lines spread across the wavelength range from the far-infrared to the vacuum-ultraviolet.

The rare-earth ions are set apart from other transition elements. The unfilled shell of $4 \mathrm{f}^{\mathrm{N}}$ electrons remains highly shielded within the closed $5 \mathrm{~s}^{2} 5 \mathrm{p}^{6}$ shells of the rare earth ion, so that the optical transitions maintain much of an atomiclike character even in a solid. This strong shielding of the $4 \mathrm{f}^{\mathrm{N}}$ electrons is in sharp contrast to transition metal $\mathrm{d}$ electrons, which are involved in chemical bonding, are thus strongly affected by the host lattice, and may show significant delocalization and mixing with electronic states of other ions in the lattice.

The actinide $5 \mathrm{f}$ electron configurations provide intermediate cases; stronger coupling of the $5 \mathrm{f}$ electrons to the lattice makes their properties more dependent on the specific host material. Most actinides are radioactive.

The optical linewidths of rare earth ions in crystals vary over a wide range depending on the temperature and other factors. Transition line shapes are described by an inhomogeneous distribution of transition frequencies having an inhomogeneous width $\Gamma_{\text {inh }}$, with each indistinguishable group or "packet" of ions having a homogeneous width $\Gamma_{\mathrm{h}}$. This line structure is illustrated schematically in Fig. 1.

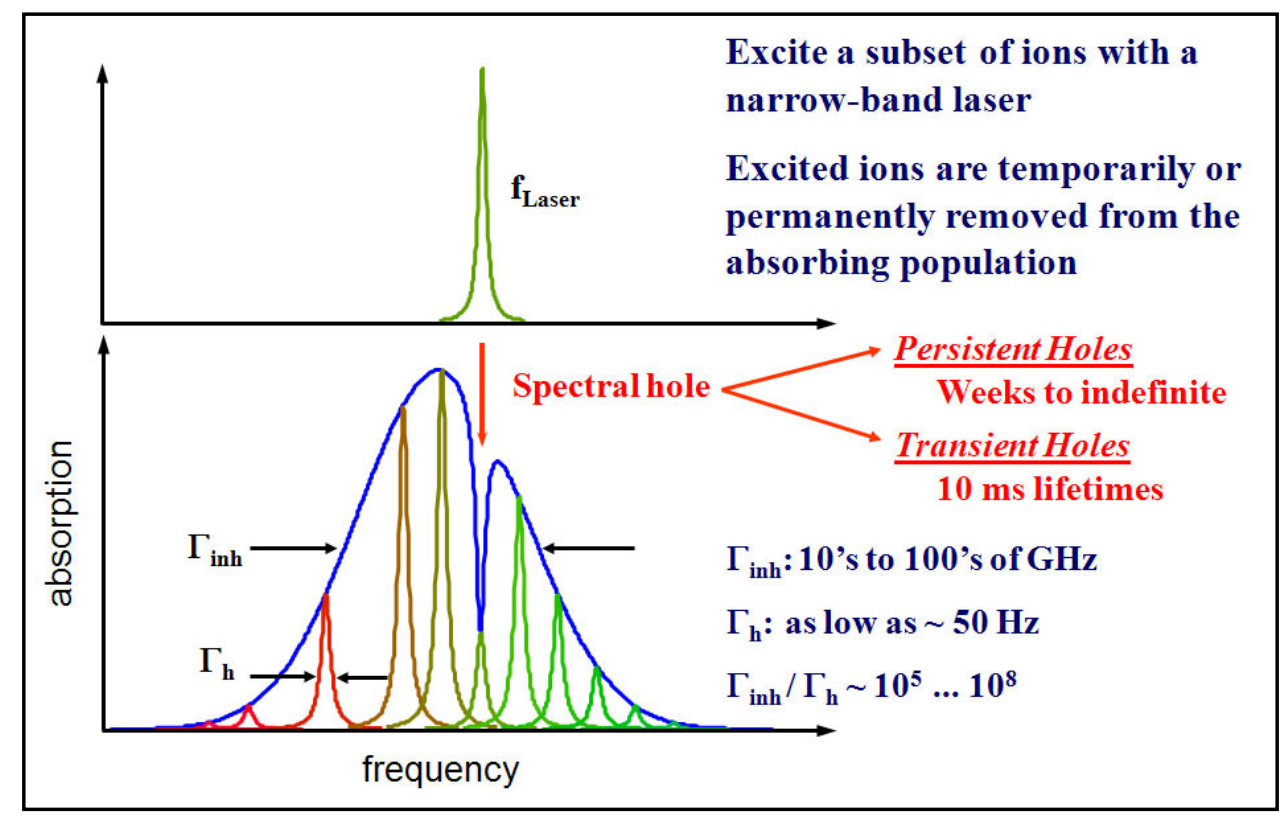

Figure 1. Illustration of the inhomogeneous linewidth $\Gamma_{\text {inh }}$ for a resonant optical material and the homogeneous linewidth $\Gamma_{\mathrm{h}}$ for individual groups of ions. Values of $\Gamma_{\mathrm{h}}$ as low as $75 \mathrm{~Hz}$ have been observed for optical transitions using two-pulse photon echo measurements. ${ }^{2-4}$ Modification of the equilibrium ground state population by absorption of light, either from a very monochromatic laser or from a complex optical waveform can produce spectral hole burning.

At room temperature, the lines are homogeneously broadened by phonons with typical homogeneous linewidths $\Gamma_{\mathrm{h}}=60$ $-1000 \mathrm{GHz}\left(2-30 \mathrm{~cm}^{-1}\right)$; stated in terms of wavelength, $\lambda / \Delta \lambda$ is on the order of $10^{4}$. As the homogeneous contribution to the linewidth is reduced by cooling, the linewidths cross over to inhomogeneous broadening in the neighborhood of $77 \mathrm{~K}$. Crystal (or glass) strain and inhomogeneity become increasingly evident at lower temperatures. Typical values of the inhomogeneous linewidth $\Gamma_{\text {inh }}$ lie in the range of $1-30 \mathrm{GHz}$ or more depending on ion concentration and crystal strain; for isolated ions, concentrations typically need to be less than $0.1 \%$ molar. When significant structural disorder is present, $\Gamma_{\text {inh }}$ can increase to greater than $100 \mathrm{GHz}$.

The narrowest homogeneous linewidths occur for transitions from the lowest component of the ground multiplet to the lowest component of excited multiplets. At low temperatures, in the range 1.5 to $10 \mathrm{~K}$, obtained by cryocoolers or by 
liquid helium, and for those "lowest-to-lowest" transitions, homogeneous linewidths $\Gamma_{\mathrm{h}}$ as narrow as tens of $\mathrm{Hz}$ to a few $\mathrm{kHz}$ can occur in favorable cases when these widths are lifetime-limited or nearly so. The narrowest observed values that we are aware of are $\Gamma_{\mathrm{h}} \sim 75-100 \mathrm{~Hz}$ observed in several crystal systems in our laboratory: $\mathrm{Er}^{3+}: \mathrm{Y}_{2} \mathrm{SiO}_{5}$ and $\mathrm{Eu}^{3+}: \mathrm{Y}_{2} \mathrm{SiO}_{5}{ }^{2-4}$

\section{MECHANISMS FOR HOMOGENEOUS LINEWIDTHS OF RARE-EARTH IONS IN INSULATORS}

At room temperature, the leading contribution to the homogeneous linewidth $\Gamma_{\mathrm{h}}$ comes from thermal phonons. Below about $4-10 \mathrm{~K}$, thermal phonon contributions are usually negligible, but to achieve this one must generally avoid systems with multiplets having small crystal field level splittings that can be bridged by low energy phonons. The phonon contribution to sharp lines is typically greater than the inhomogeneous linewidth even well below $77 \mathrm{~K}$.

Lifetime broadening makes another important contribution. The lifetime can be limited by radiative decay, non-radiative decay by spontaneous phonon emission, or other processes such as upconversion or cross-relaxation transitions involving multiple neighboring ions. In a number of cases of interest, metastable levels can be found that have a population lifetime $T_{1} \geq 1-10 \mathrm{~ms}$. A range of non-radiative decay times can occur, with many on the nanosecond or picosecond scale, though in some cases they can reach the millisecond level.

Coupling of the optical ion with fluctuating magnetic fields in its environment also contributes to the line broadening. Both nuclear and electronic spin fluctuations can contribute to these fields. If paramagnetic ions are among the dopants or undesirable impurities, their electronic magnetic moments couple strongly but are more easily frozen out with an applied magetic field. In some favorable cases, like the $\mathrm{Er}^{3+}: \mathrm{Y}_{2} \mathrm{SiO}_{5}$ and $\mathrm{Eu}^{3+}: \mathrm{Y}_{2} \mathrm{SiO}_{5}$ cited above, one may minimize and almost completely avoid magnetic interactions by composition or dilution. In other cases, an appropriate applied magnetic field can dramatically suppress these effects.

Finally there may be optically-induced changes in ion-ion coupling caused by having a neighbor of the optical ions excited by the laser light or relaxing from an excited state during the process of interest. This has been called variously 'Instantaneous Spectral Diffusion' (since it induces frequency shifts), 'excitation-induced dephasing,' 'laser-induced inhomogeneous broadening,' and 'demolition' of coherence.

\section{COMPOSITIONAL TUNING OF RARE EARTH OPTICAL TRANSITIONS}

An important consequence of the special properties of rare earth $4 \mathrm{f}^{\mathrm{N}}-4 \mathrm{f}^{\mathrm{N}}$ optical transitions is the similarity of the transition wavelengths regardless of the chemical composition of the optically-inert host material. Nevertheless, there are differences in the transition wavelengths from one material to another. Those differences are typically discrete, though we have shown together with Scientific Materials Corporation that transition wavelengths may be adjusted continuously by using mixed crystal compositions. ${ }^{5-7}$

A specific illustration ${ }^{6}$ was the development of a $944.1 \mathrm{~nm} \mathrm{Nd}^{3+}$ garnet laser for remote laser sensing of water vapor. The $\mathrm{Nd}^{3+}{ }^{4} \mathrm{~F}_{3 / 2} \rightarrow{ }^{4} \mathrm{I}_{9 / 2}$ transition occurs near $946 \mathrm{~nm}$ for the classic laser material $\mathrm{Nd}^{3+}: \mathrm{YAG}$ (yttrium aluminum garnet $\mathrm{Y}_{3} \mathrm{Al}_{5} \mathrm{O}_{12}$ ). By changing details of the crystal structure the wavelength was tuned to the desired value. Various mixtures were found to tune the $\mathrm{Nd}^{3+}$ doped mixed scandium and yttrium garnets over the 937-946 nm region. Several compositions produced the desired $944.1 \mathrm{~nm}$, allowing one to choose the one easiest to grow while optimizing the highest level of crystal perfection and other important properties. This $944 \mathrm{~nm}$ laser when frequency-doubled is coincidentally nearly optimum for transmission in ocean water.

\section{SPECTRAL HOLE BURNING AND SPECTRAL RECORDING AND PROCESSING APPLICATIONS}

Spectral hole burning (SHB) occurs when a very monochromatic laser interacts with a homogeneous "packet" of ions in these inhomogeneously broadened materials. The excited ions are temporarily or permanently removed from the absorbing population, modifying the transition frequency distribution, leaving a "hole;" this can occur by two-level saturation, optical pumping of hyperfine levels, or other physical changes. SHB materials have many capabilities that are just beginning to be appreciated by larger audiences, and those capabilities enable an array of optoelectronic devices. SHB materials can be used in combination with conventional holography to create the new powerful concepts of spectral-spatial holography (S2); S2, also called time- and space-domain holography, offers real time optical signal 
processing at bandwidths exceeding those of electronics. Spectral holes can dramatically affect optical dispersion, leading to slow light effects. In the context of ultrasound optical tomography (UOT), the narrow spectral holes provide the needed spectral filter for signal discrimination.

The properties of spectral holes in rare earth doped materials vary widely and can be tailored by choice of ion and host material and by design of material concentration and applied fields. In some materials, persistent holes are observed lasting from weeks to indefinitely or as long as the low temperatures are maintained. In other materials, transient holes, ideal for other applications, have hole lifetimes determined by radiative lifetimes and range from a few ns to $10 \mathrm{~ms}$.

In summary, typical measured values of $\Gamma_{\text {inh }}$ range from a few GHz to 100's of GHz. The homogeneous linewidth $\Gamma_{\mathrm{h}}$ can be as low as $\sim 75-100 \mathrm{~Hz}^{2-4}$ In many cases we have observed the ratio to be $\Gamma_{\text {inh }} / \Gamma_{\mathrm{h}} \sim 10^{5}-10^{8}$. 2,8-13 One, therefore, can think of these crystals as holographic recording media capable of distinguishing up to $10^{8}$ different colors. Spatialspectral holography, also called "S2," "4-d" holography, or time- and space-domain holography is a synthesis of spectral hole burning, spatial holography, and Fourier optics. This S2 holography is capable of optical signal processing, rangeDoppler radar signal processing, and spectrum analysis at high bandwidths well into the $\mathrm{GHz}$ regime and beyond the bandwidth of related electronic signal processing. It is capable of generating true-time delay for phased arrays and general correlation and convolution of optical pulse trains at multi-GHz bandwidths. To support those developments, a wide range of rare earth doped materials has been designed, developed, and studied in detail. Many of the same materials have been proven to be excellent materials for quantum information and slow light physics; examples include quantum memory for photons and demonstrations of very slow light.

An example of one of the most promising known systems for enabling a wide range of SHB applications is the resonant optical material $\mathrm{Er}^{3+}$-doped yttrium oxyorthosilicate $\left(\mathrm{Er}^{3+}: \mathrm{Y}_{2} \mathrm{SiO}_{5}\right)$ that we introduced more than a decade ago. ${ }^{3,4,14-16}$ This material system exhibits a number of important properties including unusually long coherence lifetimes, a nearly oscillator-strength-limited excited state liftetime, and operation in the $1.5 \mu \mathrm{m}$ telecommunication band where extensive low-cost optical hardware has been developed and commercially tested by the telecommunications industry. These $\mathrm{Er}^{3+}: \mathrm{Y}_{2} \mathrm{SiO}_{5}$ materials have been successfully employed in a wide range of demonstrations that include wideband signal processing, ${ }^{17}$ all-optical correlators, ${ }^{18,19}$ radio-frequency spectral analysis, ${ }^{20}$ laser frequency stabilization, ${ }^{21,22}$ electromagnetically induced transparency (EIT) ${ }^{23}$ ultraslow light propagation, ${ }^{24}$ coherent single photon storage and retrieval, ${ }^{25}$ and dynamic spectral filtering. ${ }^{1}$ The $\mathrm{Er}^{3+}: \mathrm{Y}_{2} \mathrm{SiO}_{5}$ system has also been considered and studied for its use in quantum memory applications. ${ }^{26-28}$

\section{RARE EARTH MATERIALS FOR QUANTUM MEMORIES}

We have given several reviews of rare earth materials for quantum memory and related applications. ${ }^{1,928-33}$ Examples of materials we have designed, characterized, and optimized for these applications are $\mathrm{Er}^{3+}: \mathrm{Y}_{2} \mathrm{SiO}_{5}$ at $1.5 \mu \mathrm{m}, 3,4,10,14-16,34$ $\mathrm{Er}^{3+}$-doped fibers and glasses, ${ }^{35-37} \mathrm{Tm}^{3+}: \mathrm{LiNbO}_{3}$ at $794 \mathrm{~nm},{ }^{12,13} \mathrm{Er}^{3+}: \mathrm{LiNbO}_{3}$ at $1532 \mathrm{~nm},{ }^{11}$ and $\mathrm{Eu}^{3+}: \mathrm{Y}_{2} \mathrm{SiO}_{5}$ at $580 \mathrm{~nm},{ }^{2,8,38}$ and $\mathrm{Er}^{3+}: \mathrm{Eu}^{3+}: \mathrm{Y}_{2} \mathrm{SiO}_{5}$ where the $\mathrm{Eu}^{3+}$ doping served to increase the processing bandwidth to $\sim 10 \mathrm{GHz}$ at $1.5 \mu \mathrm{m} .{ }^{39}$ The doped $\mathrm{LiNbO}_{3}$ materials are well-suited to waveguide implementations of quantum memory. ${ }^{40}$

In quantum memories, SHB can be used to prepare an ideal "artificial" lineshape for the system. The long coherence times are also important for quantum memories, along with the ability to devise $\Lambda$-systems of energy levels and to manipulate the states with electric and magnetic fields. ${ }^{28,41}$

\section{SPECTRAL HOLES AS FREQUENCY REFERENCES}

Ultra-narrow frequency references for laser frequency stabilization are another application of sharp spectral holes. ${ }^{21,22,42-}$ ${ }^{50}$ With $\Gamma_{\mathrm{h}} \sim 75-100 \mathrm{~Hz}$, milli-Hz laser frequency stability is practical. In a number of our demonstrations, the frequency-stabilization system based on SHB has demonstrated the interesting and unusual capability of providing stabilization even in cases where narrow lasers are not available to initially produce the ultra-narrow spectral holes. Stabilization to spectral holes was initially developed to provide ideal sources for S2 applications with excellent rejection of environmental perturbations due to vibration. Now manipulation and interrogation of rare earth ions used for quantum memories or in other quantum information contexts can require similarly stable lasers. More recently the group of Rosenband et al. at NIST demonstrated that stabilization to spectral holes in $\mathrm{Eu}^{3+}: \mathrm{Y}_{2} \mathrm{SiO}_{5}$ provides high performance Laser Local Oscillators for atomic clocks with environmental isolation parameters that are lower and hence better than those for optimized Fabry-Perot cavities. ${ }^{51}$ 
Frequency stabilization in this application is carried out using locking concepts involving frequency modulation spectroscopy and phase sensitive detection. Those techniques make sensitive locking with a precision of $\sim 1$ part in $10^{5}$ of the homogeneous width.

We have reported locking to both persistent and transient spectral holes, demonstrating stabilities up to a precision of a part in $10^{13}$ over milliseconds and locking to a variety of wavelengths: $\mathrm{Tm}^{3+}: \mathrm{CaF}_{2}: \mathrm{D}^{-}$at $798 \mathrm{~nm}, \mathrm{Tm}^{3+}: \mathrm{YAG}$ at $793 \mathrm{~nm}$, $\mathrm{Er}^{3+}: \mathrm{Y}_{2} \mathrm{SiO}_{5}$ at $1536 \mathrm{~nm}, \mathrm{Er}^{3+}: \mathrm{KTP}$ at $1537 \mathrm{~nm}, \mathrm{Er}^{3+}: \mathrm{CaF}_{2}: \mathrm{D}^{-}$at $1523 \mathrm{~nm}$, and $\mathrm{Er}^{3+}: \mathrm{LiYF}_{4}$ at $1530 \mathrm{~nm}^{21,22,42-50}$ Numerical modeling was also reported. ${ }^{47}$ We have given several reviews of that work. Recently Rosenband's group at NIST demonstrated laser frequency stabilization to spectral holes in $\mathrm{Eu}^{3+}: \mathrm{Y}_{2} \mathrm{SiO}_{5}$ at $580 \mathrm{~nm}$ to $6 \times 10^{-16} .{ }^{51}$

\section{LASER LINE NARROWING WITH AN SHB "DYNAMIC OPTICAL FILTER"}

A new application for SHB materials that we have recently demonstrated is for dynamic optical filtering to suppress laser phase noise and significantly "narrow" the laser linewidth. ${ }^{1}$ Lasers with very narrow spectral linewidths still exhibit a broad spectrum of phase noise limited by the relatively slow roll-off of the natural Lorentzian lineshape, even in the ideal Schawlow-Townes quantum limit. When such a laser enters a resonant SHB crystal, a spectral hole with a shape comparable to the noise power spectrum of the laser is initially burned in the absorption line. However, we have found that as the laser propagates through the resonant SHB medium, the broad phase noise of the laser is suppressed due to the nonlinear saturation of the optical transition for different spectral components of the laser. Because this frequencydependent saturation depends strongly on the power spectral density of the laser at each frequency, the medium is much more transparent at the center of the laser's spectrum compared to other frequencies where the weaker noise components may be present. Consequently, the strongest spectral component of the laser is transmitted while the noise components are partially absorbed. The spectral narrowing effect of this process also causes the spectral hole width to decrease as the light continues to propagate through the material, providing progressively greater filtering. As a result, this process is unlike other filtering methods in that it is fundamentally a spectral shaping effect due to propagation in a high optical density material with an inhomogeneously broadened absorption line.

This SHB dynamic self-filtering has a number of unique capabilities that are unmatched by any other technique. 1) While active laser stabilization typically only suppresses laser noise over a few megahertz at best, limited by the stable electronic bandwidth of PID feedback servos, SHB filtering suppresses laser noise over the entire inhomogeneous bandwidth of the material, up to hundreds of gigahertz. 2) Unlike fixed spectral filters, SHB filtering automatically matches both the frequency and spectral width of the input laser source so that it naturally follows slow changes in the laser without need for active control. 3) Very high noise suppression may be easily achieved with a high optical density material. Using $0.02 \% \mathrm{Er}^{3+}: \mathrm{Y}_{2} \mathrm{SiO}_{5}$ as an example, the peak absorption coefficient of $50 \mathrm{~cm}^{-1}$ allows a $5 \mathrm{~mm}$ thick crystal to provide more than $100 \mathrm{~dB}$ of noise suppression. 4) Under the appropriate choice of conditions, the strong nonlinearity of the hole burning process and the progressive narrowing effect of the spectral holes can be exploited to provide nearly $100 \%$ transmission through the material at the peak of the laser's power spectral density while still providing large suppression of laser noise at nearby frequencies. 5) This filtering effect is ultimately only limited by the homogeneous linewidth of the SHB material, which can be exceptionally sharp, as narrow as $73 \mathrm{~Hz}$ in $\mathrm{Er}^{3+}: \mathrm{Y}_{2} \mathrm{SiO}_{5}$. Moreover, we have demonstrated that the strong nonlinearity of the propagation process can result in filtering well below the homogeneous linewidth of the material.

An example of the SHB dynamic optical filtering effect is presented in Fig. 2 for $0.02 \% \mathrm{Er}^{3+}: \mathrm{Y}_{2} \mathrm{SiO}_{5}$ at $1536 \mathrm{~nm}$ with an applied magnetic field of $0.5 \mathrm{~T}$ along the crystal's $\mathrm{D}_{1}$ axis and a temperature of $1.6 \mathrm{~K}$. For this data, the laser noise spectrum was measured using standard delayed self-heterodyne techniques with a $24 \mu$ s optical delay line. ${ }^{52,53}$ The dotted line in Fig. 2 shows the measured self-heterodyne power spectrum of an external cavity diode laser (ECDL) with a $200 \mathrm{kHz}$ optical linewidth before passing through the crystal and the solid line shows the spectrum of the light transmitted through the crystal. The minimum noise level of $-58 \mathrm{~dB}$ observed in these measurements was due to the electronic noise floor of the photodetector. From these results, we observe that the transmitted spectrum is significantly narrowed with only a small reduction of peak optical power due primarily to reflection losses from the cryostat and other optics. For this demonstration, we specifically chose conditions where we expect a relatively broad effective homogeneous linewidth of $3.2 \mathrm{MHz}$ full-width at half maximum (FWHM), limited by spectral diffusion. ${ }^{15}$ A crystal with an optical density (OD) of 3.1 was used for this demonstration to show that the strong nonlinearity of the absorption with propagation can lead to substantial suppression of the Lorentzian tails of the laser spectrum even in a case where the homogeneous linewidth is much broader than the laser spectrum. 


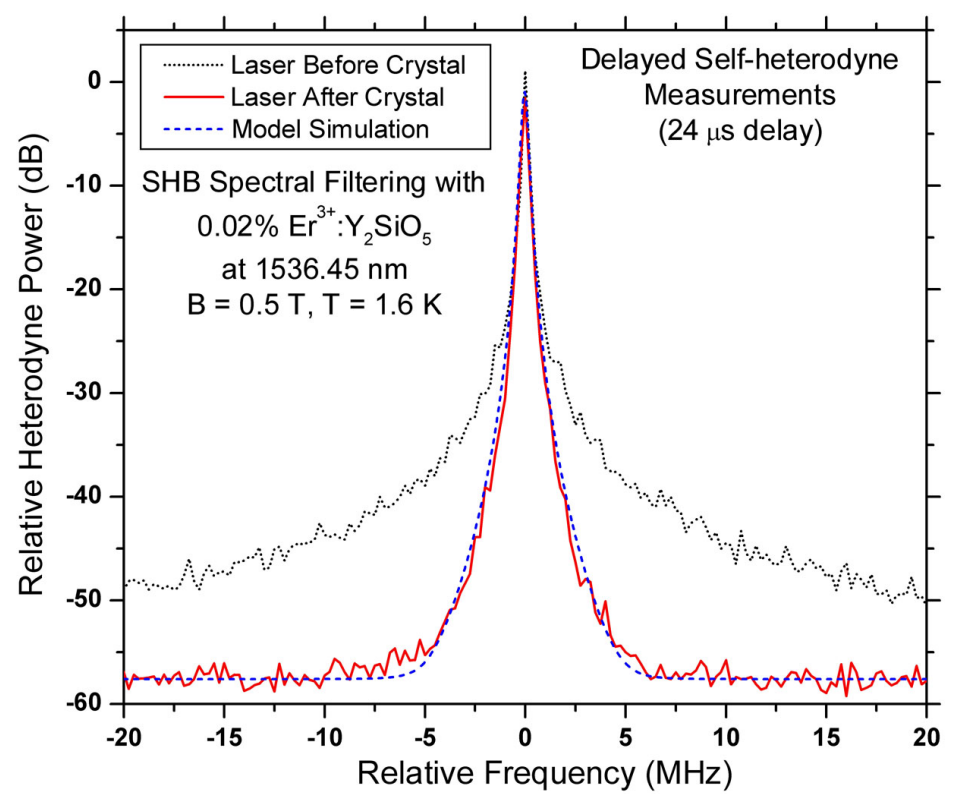

Figure 2. Example of delayed self-heterodyne measurement of the laser narrowing effect for a $200 \mathrm{kHz}$ laser before (dotted line) and after (solid line) passing through a $0.02 \% \mathrm{Er}^{3+}: \mathrm{Y}_{2} \mathrm{SiO}_{5}$ crystal with $\mathrm{OD}=3.1$ and an estimated spectral-diffusion-limited homogeneous linewidth of $3.2 \mathrm{MHz}$. A numerical simulation of the predicted SHB narrowing effect for these conditions is shown by the dashed line.

We have also carried out detailed numerical modeling of the SHB narrowing effect to understand the process and optimize the effect for different conditions. The dashed line shows a theoretical simulation of the transmitted spectrum predicted for the experimental conditions in Fig. 2, giving excellent agreement with the measured spectrum. Using this model, we have calculated expected spectral hole filter profiles that could be produced for applications such as medical imaging, as discussed in the next section. These results are shown in Fig. 3, where we have simulated the transmission spectrum of a $1.4 \mathrm{~mm}$ thick crystal of $0.02 \% \mathrm{Er}^{3+}: \mathrm{Y}_{2} \mathrm{SiO}_{5}$ at $1536 \mathrm{~nm}$ for conditions of an applied field of $5.0 \mathrm{~T}$ along the $\mathrm{D}_{1}$ axis and a temperature of $1.8 \mathrm{~K}$ where the homogeneous linewidth is only $3 \mathrm{kHz}$ and all other model

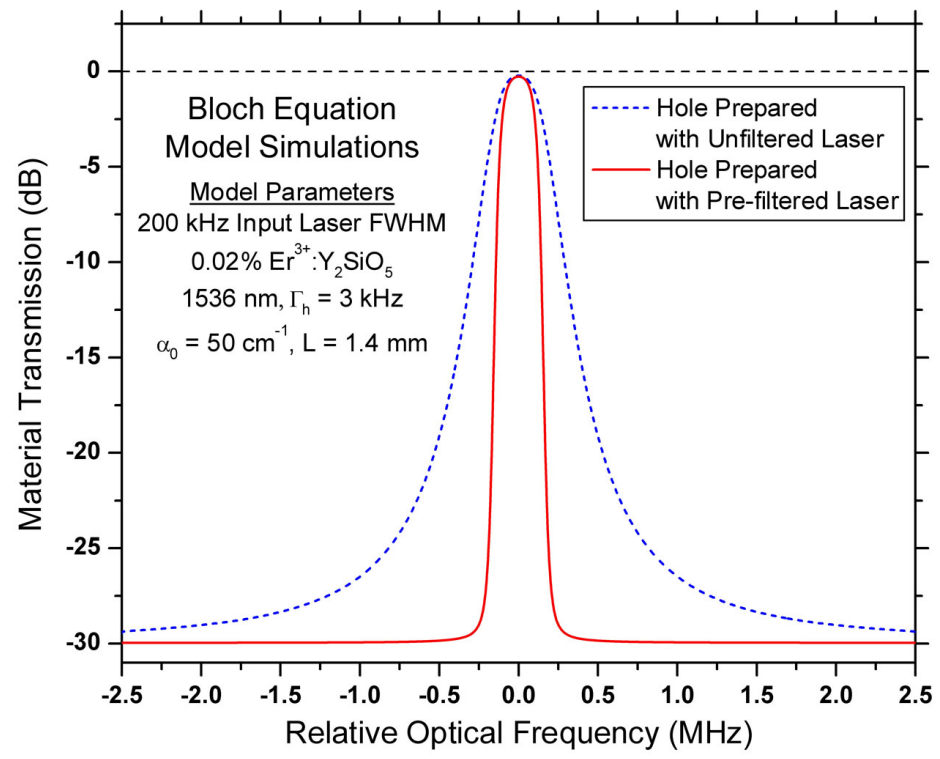

Figure 3. Numerical simulation of the transmission spectra of SHB filters with OD $=3$ and holes prepared by a $200 \mathrm{kHz}$ linewidth laser with (solid line) and without (dashed line) SHB pre-filtering, demonstrating how this method may be used to create high-contrast, low-loss optical filters with very sharp spectral edges. 
parameters are accurately known. These calculations show the expected material transmission spectrum when a laser with a natural linewidth of $200 \mathrm{kHz}$ is used to prepare the spectral hole and when the laser is pre-filtered by a separate SHB material, or even by passing through a different spot in the same crystal before burning the desired spectral hole filter. The non-Lorentzian power spectrum of the filtered laser produces a dramatically narrower spectral hole with very sharp edges while still maintaining a high transmission as compared to the hole burned by the unfiltered laser, resulting in ideal properties for medical imaging.

A demonstration of how active laser frequency stabilization may be combined with this passive filtering technique is presented in Fig. 4. For these measurements, we frequency stabilized the $200 \mathrm{kHz}$ ECDL to a high-finesse Fabry-Perot cavity with a linewidth of $100 \mathrm{kHz}$. The dotted line in Fig. 4 shows the delayed self-heterodyne measurement of the freerunning laser and the dashed line shows the frequency stabilized laser spectrum. These results are typical of active feedback control methods, where low-frequency noise is strongly suppressed resulting in a sharp peak at the optical carrier frequency while noise at frequencies beyond the bandwidth of the feedback PID servo electronics are slightly increased due to effects such as electronic noise and phase delays in the feedback correction. The solid line in Fig. 3 shows the measured spectrum of the stabilized laser after passing through a resonant $0.1 \% \mathrm{Er}^{3+}: \mathrm{LiNbO}_{3} \mathrm{SHB}_{\text {crystal }}$ with $\mathrm{OD}=2.5$ for an applied field of $5.0 \mathrm{~T}$ along the crystal's c-axis and at $1.8 \mathrm{~K}$. The sharp central peak of the power spectrum produced by the active stabilization burns a very sharp spectral hole that is very effective at eliminating the remaining laser noise. Consequently, the active stabilization and passive filtering processes perfectly complement each other to produce an exceptionally sharp laser power spectrum.

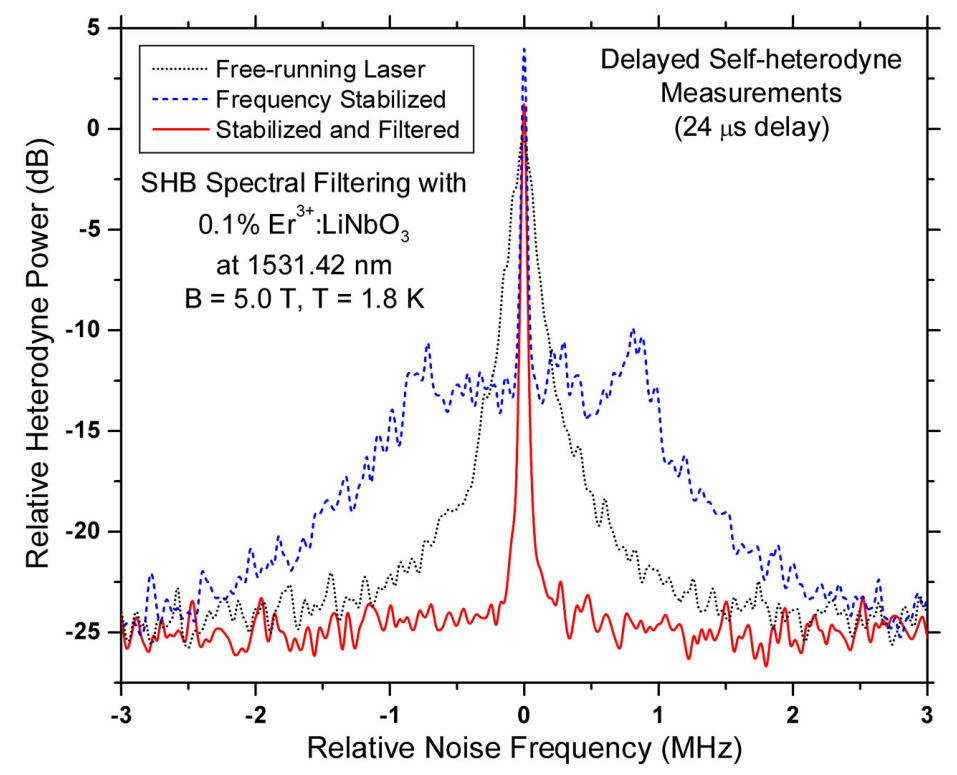

Figure 4. Example showing how active laser stabilization and passive SHB spectral filtering may be combined to produce an exceptionally sharp laser power spectrum. The dotted line is a delayed self-heterodyne measurement of a free-running $200 \mathrm{kHz}$ laser, the dashed line is the spectrum after frequency stabilizing the laser to a Fabry-Perot reference cavity, exhibiting typical noise sidebands at frequencies beyond the electronic servo bandwidth, and the solid line is the stabilized laser light after passing through a $0.1 \% \mathrm{Er}^{3+}: \mathrm{LiNbO}_{3} \mathrm{SHB}$ crystal with OD $=2.5$, demonstrating dramatic suppression of noise over all frequencies.

Together, these results demonstrate how SHB may be used as dynamic optical filters for laser sources and provide a number of unique properties beyond the capabilities of other filtering or active stabilization methods.

\section{ULTRASONIC-OPTICAL TISSUE IMAGING EXAMPLE}

One current material used for published reports of ultrasonic-optical tissue imaging (UOT) is $\operatorname{Pr}^{3+}: \mathrm{Y}_{2} \mathrm{SiO}_{5}$, which was chosen on account of strong optical absorption, $\mathrm{kHz}$ homogeneous linewidths, long hole lifetime, and the ability to burn very deep holes that burn down to near perfect transparency. This material was initially developed at MSU working together with Scientific Materials Corporation in Bozeman, MT. ${ }^{54}$ The $\mathrm{Y}_{2} \mathrm{SiO}_{5}$ host is a transparent material available in large boules from Scientific Materials; it has very high optical quality and is a laser grade material. Fabricated and 
polished parts can be supplied by Scientific Materials. Application to UOT has been carried out by the groups of Wang, Hemmer, Kröll, and others. ${ }^{55-63}$

\section{0. $\mathrm{Tm}^{3+}: \mathrm{LiNbO}_{3}$ FOR UOT TISSUE IMAGING WITH LASER LINE NARROWING "DYNAMIC OPTICAL FILTER"}

To attain the combination of properties required for UOT tissue imaging applications, we propose a new potential filtering material, $\mathrm{Tm}^{3+}: \mathrm{LiNbO}_{3}$. By combining this material with the SHB laser self-filtering technique discussed earlier, ideal optical filters may be produced that are well suited for UOT. Our detailed studies have demonstrated that this material has very strong absorption, narrow homogeneous linewidths, and long-lived persistent spectral holes. ${ }^{12,13}$

An example of a persistent spectral hole at $794 \mathrm{~nm}$ in $0.1 \% \mathrm{Tm}^{3+}: \mathrm{LiNbO}_{3}$ is shown in Fig. 5 with a small applied field of $115 \mathrm{G}$ along the crystal's c-axis and a temperature of $1.8 \mathrm{~K}$. The persistent hole burning is enabled by the nuclear hyperfine structure due to the spin $1 / 2$ nucleus of ${ }^{169} \mathrm{Tm}$. Population redistribution among the nuclear hyperfine states results in a single pair of antiholes, as seen in Fig. 5, although no sidehole structure is observed in the spectrum, unlike other system that display persistent hyperfine hole burning. The typical persistent spectral hole shown in Fig. 5 corresponds to a change in absorption of $\sim 8 \mathrm{~cm}^{-1}$, corresponding to a change in material transmission of $30 \mathrm{~dB}$ over an $8.6 \mathrm{~mm}$ thick sample for these conditions. This hole spectrum was measured 2.7 seconds after the end of the burn pulse, showing little change in depth over this timescale. To determine the spectral hole lifetime for these conditions, we measured the decay of the spectral hole area over time, as plotted in the bottom panel of Fig. 5. We observe an exponential decay with a hole lifetime of 36 seconds for these conditions.
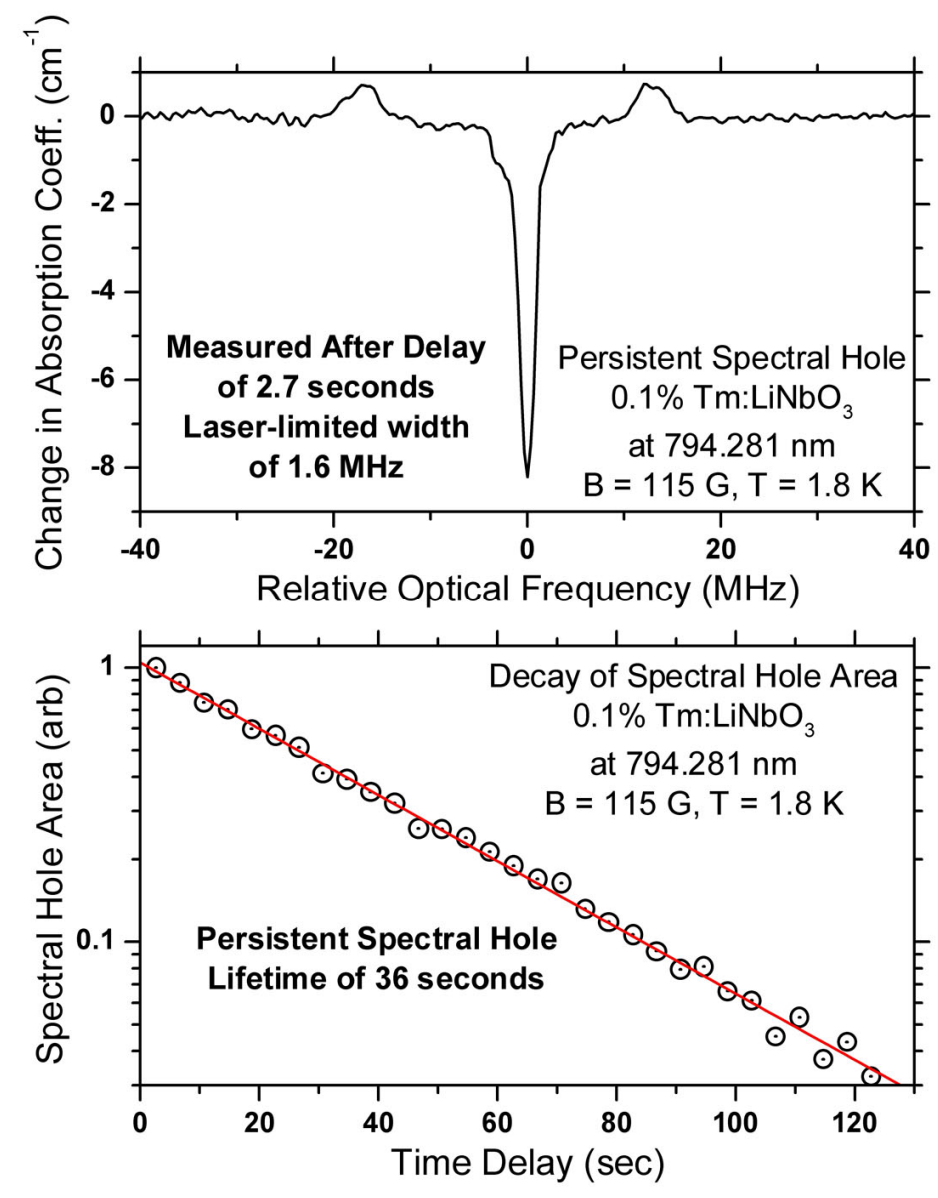

Figure 5. Top: Example of a deep persistent spectral hole at $794 \mathrm{~nm}$ in $0.1 \% \mathrm{Tm}^{3+}: \mathrm{LiNbO}_{3}$ at $1.8 \mathrm{~K}$ with an applied magnetic field of $115 \mathrm{G}$ along the crystal's c-axis. The observed hole width was limited by the laser linewidth. Bottom: Measured spectral hole decay for these conditions revealing a hole lifetime of 36 seconds. 
While we readily observed $\Delta \alpha=8 \mathrm{~cm}^{-1}$ deep holes in this material, we also burned deeper saturated spectral holes to determine the maximum filter contrast and minimum residual absorption that can be achieved for this particular position on the inhomogeneous lineshape. Under the same conditions as the data in Fig. 5, we were able to burn persistent spectral holes as deep as $11.5 \mathrm{~cm}^{-1}$. Since the absorption coefficient at this frequency is $13 \mathrm{~cm}^{-1}$, we estimate that the residual absorption at the peak of the hole is $1.5 \mathrm{~cm}^{-1}$. For a crystal $6 \mathrm{~mm}$ thick, this would correspond to a $30 \mathrm{~dB}$ filter contrast for a spectral hole with $4 \mathrm{~dB}$ of residual absorption loss through the crystal. These numbers nearly meet the application requirement of UOT, and based on our recent analysis of the optical transition structure,${ }^{13}$ we expect that the residual absorption can be significantly reduced by selecting a different position on the absorption line optimized for this application.

For the spectral hole shown in Fig. 5, the $\sim 1 \mathrm{MHz}$ linewidth of the diode laser limited the spectral resolution of the measurements to $\sim 2 \mathrm{MHz}$. This is consistent with the observed hole width of $\sim 1.6 \mathrm{MHz}$, suggesting that the intrinsic homogeneous linewidth of the material is $<1 \mathrm{MHz}$ for these conditions. To investigate this, we measured stimulated photon echo decays in this material over different timescales to determine the effective homogeneous linewidth. The maximum timescale of these measurements was $2 \mathrm{~ms}$, experimentally limited by the frequency jitter of the diode laser. An example stimulated photon echo decay at $794.267 \mathrm{~nm}$ for a $\mathrm{t}_{23}$ delay of $2 \mathrm{~ms}$ is shown in Fig. 6 with an applied field of $138 \mathrm{G}$ along the c-axis and a temperature of $1.7 \mathrm{~K}$. The exponential echo decay indicates a homogeneous linewidth of $16 \mathrm{kHz}$ under these conditions. No change in linewidth was observed over the measured timescales of $1 \mu \mathrm{s}$ to $2 \mathrm{~ms}$. Measurements as a function of temperature indicate that the homogeneous linewidth remains $<400 \mathrm{kHz}$ for temperatures of up to $6 \mathrm{~K}^{13}$ However, the persistent spectral hole lifetimes decrease with temperature to 0.5 seconds at $5.0 \mathrm{~K}$ for a field of $345 \mathrm{G},{ }^{12}$ although increasing the magnetic field strength may further increase the lifetime at these temperatures.

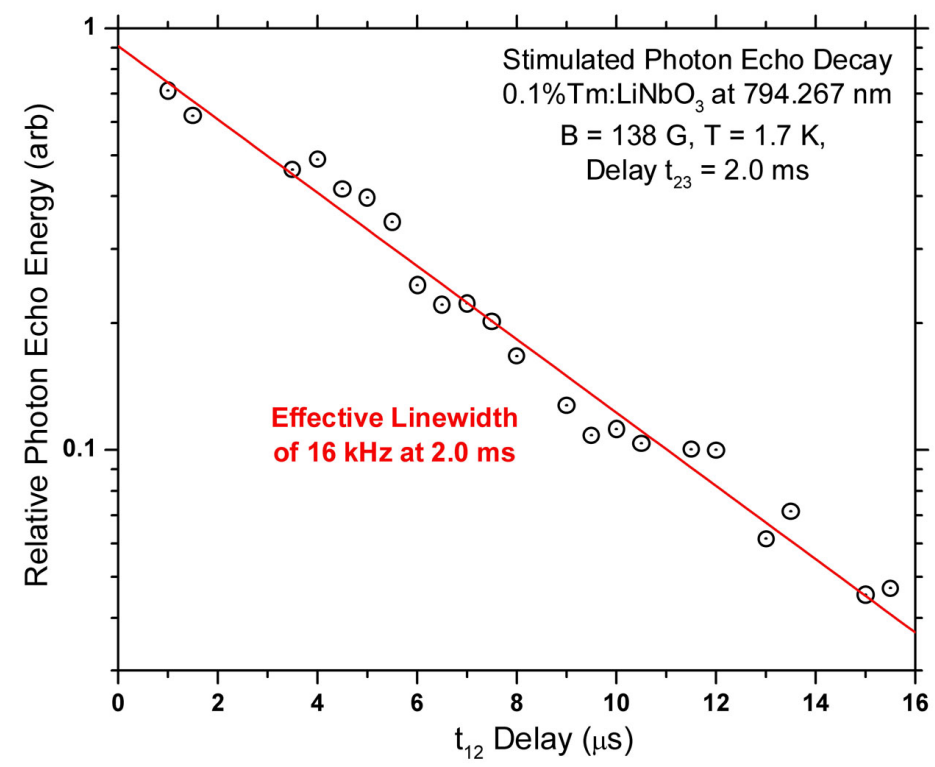

Figure 6. Measured stimulated photon echo decay curve at $794 \mathrm{~nm}$ in $0.1 \% \mathrm{Tm}^{3+}: \mathrm{LiNbO}_{3}$ for $1.7 \mathrm{~K}$ with an applied magnetic field of $138 \mathrm{G}$ along the crystal's c-axis indicating an effective homogeneous linewidth of $16 \mathrm{kHz}$ over timescales of $2 \mathrm{~ms}$.

These results suggest that $\mathrm{Tm}^{3+}: \mathrm{LiNbO}_{3}$ is one of the most promising materials to provide SHB filtering for applications such as UOT. Furthermore, since both the spatial and spectral laser beam profiles have a significant impact on the performance of SHB filters, employing SHB in the material to pre-filter the laser may further improve both the efficiency and contrast of the spectral filtering process. In our projected system, the demonstrated capability of SHB laser narrowing by an SHB dynamic optical filter is ideal to pre-filter the UOT laser. A portion of the laser can then be frequency-shifted to prepare an improved filter to be used for the UOT detection itself. Since the Lorentzian tails of a laser's natural power spectrum only roll-off at $20 \mathrm{~dB} / \mathrm{decade}$, the narrower non-Lorentzian light spectrum produced by SHB pre-filtering is ideally suited for creating the sharp high-contrast spectral holes required for efficient filtering as well as providing a more spectrally concentrated optical signal for high-sensitivity UOT signal detection. 


\section{SUMMARY AND CONCLUSIONS}

Here and in previous reviews we have described and tabulated the known properties of a range of spectral hole burning materials for applications to quantum memories, optical signal processing, laser frequency stabilization, dynamic

spectral filters, and ultrasound optical tomography. ${ }^{1,928-33}$ A wide selection of materials optimized for different properties is required to enable this entire range of applications.

The feasibility and eventual practicality of the applications and devices listed above depend on special resonant optical materials, among which rare-earth ions doped into dielectric crystals at cryogenic temperatures are one of the most promising candidates. Continued material development and understanding of the physics underlying the materials, thus needs to be given equal importance to the development of device concepts.

For UOT and related applications, the new SHB-based dynamic spectral filtering technique described here allows phase noise and side modes of the laser to be suppressed to a fraction of the achievable spectral hole width, potentially leading to both improved lasers for UOT and improved SHB-based filter preparation for signal detection.

\section{ACKNOWLEDGEMENTS}

The work presented here was supported in part by the US Air Force Research Laboratory under Agreement Nos. F49620-97-1-0411, F49620-98-1-0171, F49620-01-1-0313, andF49620-00-1-0314, the Army Research Office under contract no. W911NF-05-1-0358, and the National Science Foundation under grant no. 0903937. The crystal for the dynamic spectral filtering was grown by Scientific Materials Corp. of Bozeman, MT, USA, as were most of the other samples described in this work. Any opinions, findings, and conclusions or recommendations expressed in this material are those of the authors and do not necessarily reflect the views of the Air Force Research Laboratory, Army Research Office, or National Science Foundation.

\section{REFERENCES}

[1] Thiel, C. W., Böttger, T., and Cone, R. L., "Rare-earth-doped materials for applications in quantum information storage and signal processing," J. Lumin. 131, 353-361 (2011).

[2] Equall, R. W., Sun, Yongchen, Cone, R. L., and Macfarlane, R. M., "Ultra-slow optical dephasing in $\mathrm{Eu}^{3+}: \mathrm{Y}_{2} \mathrm{SiO}_{5}$," Phys. Rev. Lett. 72, 2179-2182 (1994).

[3] Böttger, T., Sun, Y., Thiel, C. W., and Cone R. L., "Material optimization of $\mathrm{Er}^{3+}: \mathrm{Y}_{2} \mathrm{SiO}_{5}$ at $1.5 \mu \mathrm{m}$ for optical processing, memory, and laser frequency stabilization applications," Proc. SPIE 4988, 51 (2003).

[4] Böttger, Thomas, Thiel, C. W., Cone, R. L. and Sun, Y., "Effects of magnetic field orientation on optical decoherence in $\mathrm{Er}^{3+}: \mathrm{Y}_{2} \mathrm{SiO}_{5}$," Phys. Rev. B 79, 115104 (2009).

[5] Walsh, B. M., Barnes, N. P., Hutcheson, R. L., Equall, R. W. and Di Bartolo, B., "Spectroscopy and lasing characteristics of Nd-doped $\mathrm{Y}_{3} \mathrm{Ga}_{\mathrm{x}} \mathrm{Al}_{(5-\mathrm{x})} \mathrm{O}_{12}$ materials: application toward a compositionally tuned 0.94- $\mu \mathrm{m}$ laser," JOSA B 15(11), 2794-2801 (1998).

[6] Ermeneux, F. S., Equall, R. W., Hutcheson, R. L., Cone, R. L., Moncorgé, R., Barnes, N. P., Gallagher, H. G., and Han, T. P., "Nd-doped scandium garnets for compositional tuning to $944.1 \mathrm{~nm}$ and improved laser performance around 945 nm", [OSA Trends in Optics and Photonics Advanced Solid State Lasers 1999, Vol. 26, edited by Fejer, M. M., Injeyan, H., and Keller, U.] Optical. Society of America, Washington, DC, 242-248 (1999).

[7] Braud, A., Ermeneux, F. S., Sun, Y., Cone, R. L., Equall, R. W., Hutcheson, R. L., Maunier, C., Moncorgé, R., Barnes, N. P., Gallagher, H. G., and Han, T. P., "Nd-Doped Mixed Scandium Garnets for Improved Laser Performance and Compositional Tuning From 937 to 946 nm," [OSA Trends in Optics and Photonics on Advanced Solid State Lasers 2001, Vol. 50, edited by Marshall, C.] Optical. Society of America, Washington, DC, 265-269 (2001).

[8] Könz, Flürin, Sun, Y., Thiel, C. W., Cone, R. L., Equall, R. W., Hutcheson, R. L., and Macfarlane, R. M., "Temperature and concentration dependence of optical dephasing, spectral hole lifetime, and anisotropic absorption in $\mathrm{Eu}^{3+}: \mathrm{Y}_{2} \mathrm{SiO}_{5}$, , Phys. Rev. B 68, 085109 (2003). 
[9] Sun, Y. C., "Rare earth materials in optical storage and data processing applications," [Spectroscopic Properties of Rare Earths in Optical Materials], edited by Liu, Guokui, and Jacquier, B. Springer, Berlin and Tsingua University Press, 373-424 (2005).*-

[10] Böttger, Thomas, Thiel, C. W., Cone, R. L., and Sun, Y., "Controlled compositional disorder in $\mathrm{Er}^{3+}: \mathrm{Y}_{2} \mathrm{SiO}_{5}$ for wide bandwidth hole burning material at $1.5 \mu \mathrm{m}$," Phys. Rev. B 77, 155125 (2008).

[11] Thiel, C. W., Macfarlane, R. M., Böttger, T., Sun, Y., Cone, R. L., and Babbitt, W. R., "Optical decoherence and persistent spectral hole burning in $\mathrm{Er}^{3+}: \mathrm{LiNbO}_{3}$, J. Lumin. 130, $1603-1609$ (2010).

[12] Thiel, C. W., Sun, Y., Böttger, T., Babbitt, W. R., and Cone, R. L., "Optical decoherence and persistent spectral hole burning in $\mathrm{Tm}^{3+}: \mathrm{LiNbO}_{3}$, J. Jumin. 130, 1598 - 1602 (2010).

[13] Sun Y., Thiel C. W., and Cone R. L., "Optical decoherence and energy level structure of $0.1 \% \mathrm{Tm}^{3+}: \mathrm{LiNbO}_{3}$," Submitted to Phys. Rev. B. (2012).

[14] Macfarlane, R. M., Harris, T. L., Sun, Y., Cone, R. L. and Equall, R. W., "Measurement of Photon Echoes at 1.5 microns in $\mathrm{Er}^{3+}: \mathrm{Y}_{2} \mathrm{SiO}_{5}$ using a diode laser and amplifier," Opt. Lett. 22, 871-873 (1997).

[15] Böttger, Thomas, Thiel, C. W., Sun, Y., and Cone, R. L., "Optical decoherence and spectral diffusion at $1.5 \mu \mathrm{m}$ in $\mathrm{Er}^{3+}: \mathrm{Y}_{2} \mathrm{SiO}_{5}$ versus magnetic field, temperature, and $\mathrm{Er}^{3+}$ concentration," Phys. Rev. B 73, 075101 (2006).

[16] Böttger, Thomas, Thiel, C. W., Sun, Y. and Cone, R. L., "Spectroscopy and dynamics of $\mathrm{Er}^{3+}: \mathrm{Y}_{2} \mathrm{SiO}_{5}$ at $1.5 \mu \mathrm{m}$," Phys. Rev. B 74, 075107 (2006).

[17] Cole, Z., Böttger, T., Krishna Mohan, R., Reibel, R., Babbitt, W. R., Cone, R. L. and Merkel, K. D., "Coherent integration of $0.5 \mathrm{GHz}$ spectral holograms at $1536 \mathrm{~nm}$ using dynamic bi-phase codes," Appl. Phys. Lett. 81, 3525 (2002).

[18] Harris, T. L., Sun, Y., Cone, R. L., Macfarlane, R. M. and Equall, R. W., "Demonstration of Real-Time Address Header Decoding for Optical Data Routing at $1536 \mathrm{~nm}$," Opt. Lett. 23, 636 (1998).

[19] Harris, T. L., Sun, Y., Cone, R. L., Babbitt, W. R., Ritcey, J. A. and Equall, R. W., "Spatial-spectral holographic correlator at $1536 \mathrm{~nm}$ using 30-symbol quadriphase- and binary-phase-shift keyed codes," Opt. Lett. 25, 85 (2000).

[20] Crozatier, V., Lavielle, V., Bretenaker, F., Le Gouët, J.-L. and Lorgeré, I., "High-resolution radio frequency spectral analysis with photon echo chirp transform in an Er:YSO crystal," IEEE J. Quant. Electr. 40, 1450 (2004).

[21] Sellin, P. B., Strickland, N. M., Böttger, T., Carlsten, J. L., and Cone, R. L., "Laser stabilization at $1536 \mathrm{~nm}$ using regenerative spectral hole burning,” Phys. Rev. B 63, 155111-1 - 155111-7 (2001).

[22] Böttger, Thomas, Pryde, G. J., and Cone, R. L., "Programmable sub-kHz laser frequency stabilization at $1523 \mathrm{~nm}$ using persistent spectral hole burning," Opt. Lett. 28, 200-202 (2003).

[23] Baldit, E., Bencheikh, K., Monnier, P., Briaudeau, S., Levenson, J. A., Crozatier, V., Lorgeré, I., Bretenaker, F.,Le Gouët, J.-L., Guillot-Noël, O. and Goldner, Ph., "Identification of $\Lambda$-like systems in $\mathrm{Er}^{3+}: \mathrm{Y}_{2} \mathrm{SiO}_{5}$ and observation of electromagnetically induced transparency," Phys. Rev. B 81, 144303 (2010).

[24] Baldit, E., Bencheikh, K., Monnier, P., Levenson, J. A. and Rouget V., "Ultraslow Light Propagation in an Inhomogeneously Broadened Rare-Earth Ion-Doped Crystal," Phys. Rev. Lett. 95, 143601 (2005).

[25] Lauritzen, B., Minář, J., de Riedmatten, H., Afzelius, M., Sangouard, N., Simon, C. and Gisin, N., "Telecommunication-Wavelength Solid-State Memory at the Single Photon Level," Phys. Rev. Lett. 104, 080502 (2010).

[26] Guillot-Noël, O., Goldner, Ph., Le Du, Y., Baldit, E., Monnier, P. and Bencheikh, K., "Hyperfine interaction of $\mathrm{Er}^{3+}$ ions in $\mathrm{Y}_{2} \mathrm{SiO}_{5}$ : An electron paramagnetic resonance spectroscopy study," Phys. Rev. B 74, 214409 (2006).

[27] Hastings-Simon, S. R., Lauritzen, B., Staudt, M. U., van Mechelen, J. L. M., Simon, C., de Riedmatten, H., Afzelius, M. and Gisin, N., "Zeeman-level lifetimes in $\mathrm{Er}^{3+}: \mathrm{Y}_{2} \mathrm{SiO}_{5}$," Phys. Rev. B 78, 085410 (2008).

[28] Tittel, W., Afzelius, M., Chanelière, T., Cone, R. L., Kröll, S., Moiseev, S. A., and Sellars, M., "Photon-echo quantum memory in solid state systems," Laser \& Photon. Rev. 4, 244-267 (2010).

[29] Macfarlane, R. M., and Shelby, R. M., "Coherent transient and holeburning spectroscopy of rare earth ions in solids," [Spectroscopy of Solids containing Rare Earth Ions], edited by A. A. Kaplyanskii and R. M. Macfarlane, North Holland, Amsterdam, 51-184 (1987).

[30] Sun, Y., Thiel, C. W., Cone, R. L., Equall, R. W. and Hutcheson, R. L., "Recent progress in developing new rare earth materials for hole burning and coherent transient applications," J. Lumin. 98, 281 (2002).

[31] Macfarlane, R. M., "High-resolution laser spectroscopy of rare-earth doped insulators: a personal perspective," J. Lumin. 100, 1 (2002).

[32] Rebane, A. K., Thiel, C. W., Mohan, R. K. and Cone, R. L., "Slow decoherence and the radiative decay limit in rare-earth-doped crystals for coherent optical storage,” Bull. Russian Acad. Science: Physics 74, (2010) 934. 
[33] Rebane, A. K., Thiel, C. W., Mohan, R. K., and Cone, R. L., "Maximum coherence in optical transitions in rareearth-ion-activated solids," Proceedings of the SPIE, v 7611, 76110H (2010).

[34] Sun, Y., Böttger, Thomas, Thiel, C. W., and Cone, R. L., "Magnetic g-tensors for the ${ }^{4} \mathrm{I}_{15 / 2}$ and ${ }^{4} \mathrm{I}_{13 / 2}$ states of $\mathrm{Er}^{3+}: \mathrm{Y}_{2} \mathrm{SiO}_{5}$, , Phys. Rev. B 77, 085124 (2008).

[35] Macfarlane, R. M., Sun, Y., Sellin, P. B., and Cone, R. L., "Optical decoherence in $\mathrm{Er}^{3+}$-doped silicate fiber: evidence for coupled spin-elastic tunneling systems," Phys. Rev. Lett. 96, 033602 (2006).

[36] Sun, Y., Cone, R. L., Bigot, L., and Jacquier, B., "Exceptionally narrow homogeneous linewidth in erbium-doped glasses," Opt. Lett. 31, 3453-3455 (2006).

[37] Macfarlane, R. M., Sun, Y., Sellin, P. B., and Cone, R. L., "Optical decoherence times and spectral diffusion in an Er-doped optical fiber measured by two-pulse echoes, stimulated photon echoes, and spectral hole burning," J. Lumin. 127, 61-64 (2007).

[38] Lauritzen, B., Timoney, N., Gisin, N., Afzelius, M., de Riedmatten, H., Sun, Y., Macfarlane, R. M., and Cone, R. L., "Spectroscopic investigations of $\mathrm{Eu}^{3+}: \mathrm{Y}_{2} \mathrm{SiO}_{5}$ for quantum memory applications," Submitted to Phys. Rev. B (2011).

[39] Thiel, C. W., Babbitt, W. R. and Cone, R. L., "Optical decoherence studies of yttrium oxyorthosilicate $\left(\mathrm{Y}_{2} \mathrm{SiO}_{5}\right)$ co-doped with $\mathrm{Er}^{3+}$ and $\mathrm{Eu}^{3+}$ for optical signal processing and quantum information applications at 1.5 microns," Submitted to Phys. Rev. B (2011).

[40] Saglamyurek, E., Sinclair, N., Jin, J., Slater, J. A., Oblak, D., Bussières, F., George, M., Ricken, R., Sohler, W. and Tittel, W., "Broadband waveguide quantum memory for entangled photons," Nature 469, 512 (2011).

[41] Simon, C., Afzelius, M., Appel, J., de la Giroday, A. B., Dewhurst, S. J., Gisin, N., Hu, C., Jelezko, F., Kröll, S., Müller,J. H., Nunn, J., Polzik, E., Rarity, J., de Riedmatten, H., Rosenfeld, W., Shields, A. J., Sköld, N., Stevenson, R. M., Thew, R., Walmsley, I., Weber, M., Weinfurter, H., Wrachtrup, J. and Young, R. J., "Quantum Memories," Eur. Phys. J. D 58, 1 (2010).

[42] Strickland, N. M., Cone, R. L., and Macfarlane, R. M., "Persistent spectral hole burning in deuterated $\mathrm{CaF}_{2}: \mathrm{Tm}^{3+}$," Phys. Rev. B 59, 14328-14335 (1999).

[43] Sellin, P. B., Strickland, N. M., Carlsten, J. L., and Cone, R. L., "Programmable frequency reference for sub-kHz laser stabilization using persistent spectral hole burning," Opt. Lett. 24, 1038-1040 (1999).

[44] Strickland, N. M., Sellin, P. B., Sun, Y., Carlsten, J. L., and Cone, R. L., "Laser frequency stabilization using regenerative spectral hole burning," Phys. Rev. B 62, 1473-1476 (2000).

[45] Cone, R. L., Böttger, T., Pryde, G. J., Strickland, N.M., Sun, Y., Sellin, P. B., and Carlsten, J. L., "Semiconductor lasers stabilized to spectral holes in rare earth crystals," Proc. SPIE 4283, 335-346 (2001).

[46] Böttger, Thomas, Sun, Y., Pryde, G.J., Reinemer, G., and Cone, R.L., "Diode laser frequency stabilization to transient spectral holes and spectral diffusion in $\mathrm{Er}^{3+}: \mathrm{Y}_{2} \mathrm{SiO}_{5}$ at $1536 \mathrm{~nm}$," J. Lumin. 94-95, 565-568 (2001).

[47] Pryde, G. J., Böttger, T., and Cone, R. L., "Numerical modeling of laser stabilization by regenerative spectral hole burning," J. Lumin. 94-95, 587-591 (2001).

[48] Böttger, Thomas, Pryde, Geoffrey J., Strickland, Nicholas M., Sellin, Peter B., and Cone, Rufus L., "Semiconductor lasers stabilized to spectral holes in rare-earth crystals," Optics \& Photonics News, 12 (12), 23 (2001).

[49] Pryde, G. J., Böttger, T., Cone, R. L., and Ward, R. C. C., "Semiconductor lasers stabilized to spectral holes in rare earth crystals to a part in $10^{13}$ and their application to devices and spectroscopy," J. Lumin. 98, 309-315 (2002).

[50] Böttger, Thomas, Pryde, G. J., Thiel, C. W., and Cone, R. L., "Laser frequency stabilization at 1.5 microns using ultranarrow inhomogeneous absorption profiles in $\mathrm{Er}^{3+}: \mathrm{LiYF}_{4}$," J. Lumin. 127, 83-88 (2007).

[51] Thorpe, Michael J., Rippe, Lars, Fortier, Tara M., Kirchner, Matthew S., and Rosenband, Till, "Frequency stabilization to $6 \times 10^{-16}$ via spectral-hole burning," Nature Photonics 5, 688-693 (2011).

[52] Okoshi, T., Kikuchi, K., and Nakayama, A., "Novel method for high resolution measurement of laser output spectrum," Electron. Lett. 16, 630 (1980).

[53] Richter, L. E., Mandelberg, H. I., Kruger, M. S., and Mcgrath, P. A., "Linewidth Determination from SelfHeterodyne Measurements with Subcoherence Delay Times,” IEEE J. Quantum Electron. QE-22, 2070 (1986).

[54] Equall, R. W., Cone, R. L., and Macfarlane, R. M., "Homogeneous broadening and hyperfine structure of optical transitions in $\operatorname{Pr}^{3+}: \mathrm{Y}_{2} \mathrm{SiO}_{5}$," Phys. Rev. B 52, 3963-3969 (1995).

[55] Li, Y. Z., Kim, C., Zhang, H. L., Wagner, K. H., Hemmer, P. and Wang, L. V., "Pulsed ultrasound-modulated optical tomography using spectral hole-burning," Proc. SPIE 6856, 68561R (2008).

[56] Li, Y. Z., Hemmer, P., Kim, C., Zhang, H., and Wang, L. V. "Detection of ultrasound-modulated diffuse photons using spectral-hole burning," Opt. Express 16, 14862-14874 (2008). 
[57] Li, Y. Z., Zhang, H. L., Kim, C., Wagner, K. H., Hemmer, P., and Wang, L. V., "Pulsed ultrasound-modulated optical tomography using spectral-hole burning as a narrowband spectral filter," Appl. Phys. Lett. 93, 011111-1 011111-2 (2008).

[58] Tay, J. W., Ledingham, P. M., and Longdell, J. J., "Coherent optical ultrasound detection with rare-earth ion dopants," Appl. Opt. 49, 4331-4334 (2010).

[59] Xu, X., Kothapalli, S.-R., Liu, H. L., and Wang, L. V, "Spectral hole burning for ultrasound-modulated optical tomography of thick tissue,", J. Biomed. Opt. 15, 066018 (2010).

[60] Xu, X., Liu, H. and Wang, L. V., "Improving the imaging ability of ultrasound-modulated optical tomography with spectral-hole burning," Proc. SPIE 7948, 794803 (2011).

[61] Louchet-Chauvet, A., Lauro, R., Goldner, P., Ramaz, F., Chanelière, T., and Le Gouët, J-L., "High-resolution large dynamic range spectral filtering at $800 \mathrm{~nm}$ using Tm:YAG crystals," Proc. SPIE 7948, 794805 (2011).

[62] Tay, J. W., Ledingham, P.M., Longdell, J.J., "Coherent detection of ultrasound using spectral hole burning media," Proc. SPIE 7948, 794809 (2011).

[63] Zhang, H. L., Sabooni, M., Rippe, L., Kim, C., Kröll, S., Wang, L. V., Hemmer, P. R. "Slow light and ultrasound for quantum limited deep tissue imaging," submitted for publication. 